INTERNATIONAL DESIGN CONFERENCE - DESIGN 2018

https://doi.org/10.21278/idc.2018.0405

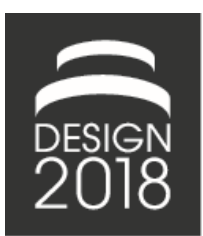

\title{
THE ROLE OF USER-CENTRED DESIGN IN SMART WEARABLE SYSTEMS DESIGN PROCESS
}

\author{
L. Francés-Morcillo, P. Morer-Camo, M. I. Rodríguez-Ferradas and A. Cazón-Martín
}

\begin{abstract}
Wearable electronics make possible to monitor human activity and behaviour. Most of these devices have not taken into account human factors and they have instead focused on technological issues. This fact could not only affect user acceptance and user experience but also the devices' use cycle. This leads us to formulate our research question: How can a user-centred design process deal with this situation? This study answers this question by reviewing the design requirements found in the literature and providing a map of design requirements to design wearable devices.
\end{abstract}

Keywords: ergonomics, human centred design, wearable, design methodology, product design

\section{Introduction}

Wearable electronics have become a recurrent technology for solving concrete problems in different fields such as medicine, leisure, sports etc. These devices make it possible to monitor human activity and behaviour, so they provide essential data for dealing with specific human needs. Although high tech approaches have been developed in wearable technologies, most of them have a short life cycle when they are launched to the marketplace and user acceptance is still not as widespread as expected. Why do these high tech devices have such life cycle problems?

In contrast to other technological devices, such as laptops and smartphones, wearables live on people's bodies. This fact involves a wholesale change in the way they should be designed. The creation of wearables requires specific concepts, techniques and ingredients involving textile, electronics and software that consider the diversity of potential users and their environments (Duval and Hoareau, 2010). Thus, successful wearable usability is no longer about providing technical success, but rather about creating an optimal user experience (Chae et al., 2007).

Furthermore, wearable systems are composed of complex parts and they have high costs in real work scenarios. Most of these devices have not taken into account human factors and they have instead focused on technological issues (Motti and Caine, 2014). This fact could not only affect user acceptance and user experience but also the devices' use cycle.

This subsequently leads us to formulate the following research question: How can a user-centred design process deal with this situation? The aim of this study is to answer this question by (1) reviewing the design requirements found in the literature, and (2) providing a list of design requirements gathering user-centered tools and techniques to design wearable devices. By meeting those two objectives, this article aims to be a first step towards wearable design based on human factors. 


\subsection{Background: Wearable definitions and design principles found in the literature}

\subsubsection{Wearable definitions}

The term 'wearable' has been defined in different ways through the years. According to Cambridge Dictionary, "Wearable technology consists of things that can be worn, such clothing or glasses, that contain computer technology or have the ability to connect to the internet" (Cambridge Dictionary, 2017).

According to this definition, there are two important requirements that need to be fulfilled in designing wearables: connectivity and wearability.

As wearables live on people's bodies, not only should they meet technological requirements, but humanfactors must also be considered in the early design stage. Thus, it is important to go deeper into the definition of wearables and provide basic design principles for wearability by gathering human-factor related parameters (Motti and Caine, 2014).

There are articles that have created tools and guidelines for wearable device design with a bio-design focus based on engineering as well as on aspects of psycho-physical well-being (Canina, 2011). There are others that propose methodologies for assessing the effects of wearables in terms of physiological energy expenditure and biomechanical effects. (Knight et al., 2007)

In terms of this user-based view, some researchers believe that a wide variety of sources should be considered in defining the issues that should be taken under consideration in academic research on wearables (Chan et al., 2012). Within this view, a complete understanding of a wearable within the context and the purpose for which it has been created for can be gained. Wearables can also be defined through a Universal Design (UD) approach in order to use the advantages of applying UD principles to different issues that wearable technologies address (Tomberg et al., 2015).

As there are multiple definitions of what a wearable device is depending on the specific focus of the study, this study has taken the following as a reference model: "A wearable is a fully functional, selfpowered, self-contained computer that is worn on the body, providing access to and interaction with information anywhere and at any time" (Wade, 2010). This definition is not based on a particular view but instead gathers general characteristics. Thus, the rest of definitions can be understood within this general one.

\subsubsection{Wearable design requirements}

As occurs with the term wearable, design requirements for wearables have also been defined in different ways. In this section, seven studies that have considered human-factors for the definition of such requirements are discussed. These studies are the only ones found in literature that show the importance of human factors and that suggest a list of design requirements focused on wearable devices.

Some studies have based the requirements definition on a particular focus, such as Maslow's hierarchy of needs: Maslow's theory provides a clear and strong base to identify and prioritize services and design worldwide from a human point of view (Duval and Hoareau, 2010). Through this point of view, (Cho et al., 2009) define six different human aspects that should be considered in the design of wearable systems: usability, functionality, durability, safety, comfort and fashion.

Other studies have considered wearability and have defined principles that should be incorporated during the design phase of the wearable creation process (Motti and Caine, 2014). For devices that will be worn on the body, other studies have proposed a methodology for assessing the effects of wearing a computer in terms of physiological energy expenditure, biomechanical effects, discomfort due to musculoskeletal loading and perceptions of well-being through comfort assessment (Knight et al., 2007). Other authors have addressed the importance of considering not only engineering aspects but also the psycho-physical well-being of users (Canina, 2011). Others studies have expressed the need to take a human-centred approach to improve interaction and to make wearables more effective, reliable, safe and engaging (Contreras-Vidal et al., 2015). The application of Universal Design principles to wearables also helps move the approach to design closer to human factors (Tomberg et al., 2015). Other studies highlighted the non-intrusive monitoring paradigm in order to do not affect user behaviour and being completely transparent to him/her own daily activities (Andreoni et al., 2016) 


\section{Methodology}

In order to get a wearable that considers the final user, it is important to follow a methodology based on human factors. We used the three-phase approach below to develop this case of wearables design presented here.

1. Setting a base. As in other works, the analysis of design requirements follows a reference model, which helps to determine the categorization of the selected requirements. Our study is based on ergonomics and human factors, taking the book of Evaluation of Human Work as our reference model (Sharples, 2014).

2. Design requirements classification. Based on ergonomics, a mind-map with all the requirements from the literature was created and categorized: physical ergonomics, cognitive ergonomics and emotional ergonomics.

3. Requirements analysis. Due to the large amount of data found in the literature, a specific methodology was defined for carrying out the requirements analysis. This requirement analysis had two steps:

a. Quantitative analysis: The list of requirements found in the literature resulted in 53 items. Thus, a quantitative analysis was done in order to be able to deal with all the information. In this analysis, different parameters were defined as quantifying parameters. This will be explained deeply in the pertinent section.

b. Qualitative analysis: To establish connections between different requirements, some usercentred design tools were used. Those techniques were based on the Symmetric and Asymmetric Clustering Matrix (Kumar, 2012).

\subsection{Ergonomics/human factors as a base for the methodology}

In terms of ergonomics-related information, while a great deal of information about software and hardware can often be found, only a few studies introduce ergonomic aspects in their design process.

We began by taking an existing definition of ergonomics as a reference model. This reference model was used to categorize design requirements into different domains of ergonomics. Although many studies on ergonomics exist, only a few of them integrate human factors into a design process for wearables. The book Evaluation of Human Work (Sharples, 2014) is a good example of adapting human factors to new processes and contexts. Based on the definition of ergonomics within four domains (physical, cognitive, social, and system ergonomics), (Sharples, 2014) presents the interconnections between those terms.

Figure 1 shows the interconnection in which three different circles that represent physical, cognitive and social ergonomics interact with each other inside a larger circle that represents system ergonomics. In spite of being separate, they are intrinsically connected to each other since non-compliance in one would directly affect the others (e.g. the lack of comfort in one product could affect a user's decision or satisfaction). Thus, it is important to take a holistic approach to ergonomics in the design process of wearable services in order to guarantee good system operation.

This perspective is applicable to wearables since their design requires considering aspects within physical, cognitive and emotional ergonomics. Firstly, as mentioned before, wearables live on people's body, so aspects related to physical ergonomics are essential for assuring the wearability and comfort of the device. Secondly, wearables have a high degree of interaction with users, so cognitive process of people are fully involved to ensuring users' needs are met. Those aspects are studied within cognitive ergonomics. Finally, the devices should solve specific problems for users and satisfy them, and in so doing increase the likelihood that users will accept the devices; all of these aspects are considered to be part of emotional ergonomics.

Given the nature of wearables, the holistic approach to ergonomics is essential for classifying wearable design requirements and placing them in the whole design process. The following section represents the design requirements found in the literature and classifies them into ergonomic specialisms. 


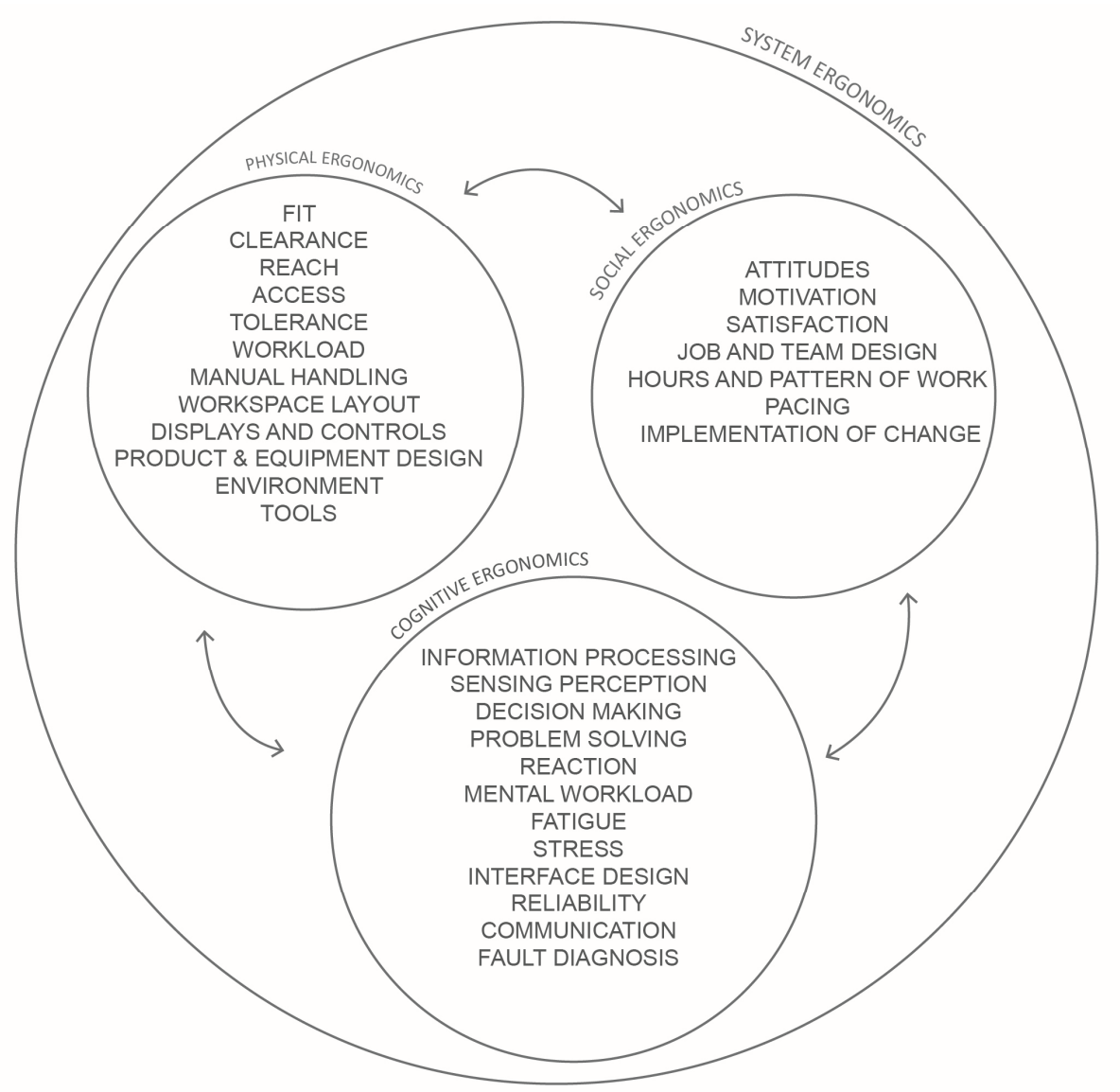

Figure 1. Ergonomic wheel system model based on (Sharples, 2014) definition of ergonomics

\subsection{Requirements visualization}

Following to the base set above, a mind-map was drawn. This mind-map shows a list of proposed design requirements divided into three groups: physical, cognitive and emotional ergonomics. This list of proposed design requirements was gathered from seven studies that analysed the role of human-factors in wearables design, in order to look for shared definitions among all.

As Figure 2 shows, all the requirements are represented in terms of physical, cognitive or social ergonomics. Although all the studies considered human-factors, only a few of them considered requirements that fall within all three domains. Most of the design requirements found in these studies were related to the physical ergonomics, but only a few of them were about cognitive and social ergonomics. This means there is a lack of cohesion in the system and that not all the design cycle has been considered.

As far as design requirements are concerned, the study resulted in a list of 52 different requirements. Looking at the number of requirements within each group it can be seen that there are many more requirements included in physical ergonomics (30/52) than in cognitive ergonomics (15/52) or emotional ergonomics (7/52).

This fact has nothing to do with their importance, but it may be related to the ease of measuring such requirements. Moreover, each group is not independent but rather they are intrinsically related to each other. That is, non-compliance of any requirement in a group would directly affect the other groups (e.g. the lack of comfort in one product could affect a user's decision making or satisfaction).

Moreover, the different terms used interferes with the analysis of each requirement's weight. In the case of the term "comfort", some studies consider only physical ergonomics but others include aspects such 
as "emotion" (Knight et al., 2007), so the use of the word is deeper in some cases than in others. This leads to the conclusion that some works use the same term to refer to different concepts. This make it impossible to draw conclusions about the most popular requirements without a specific methodology.

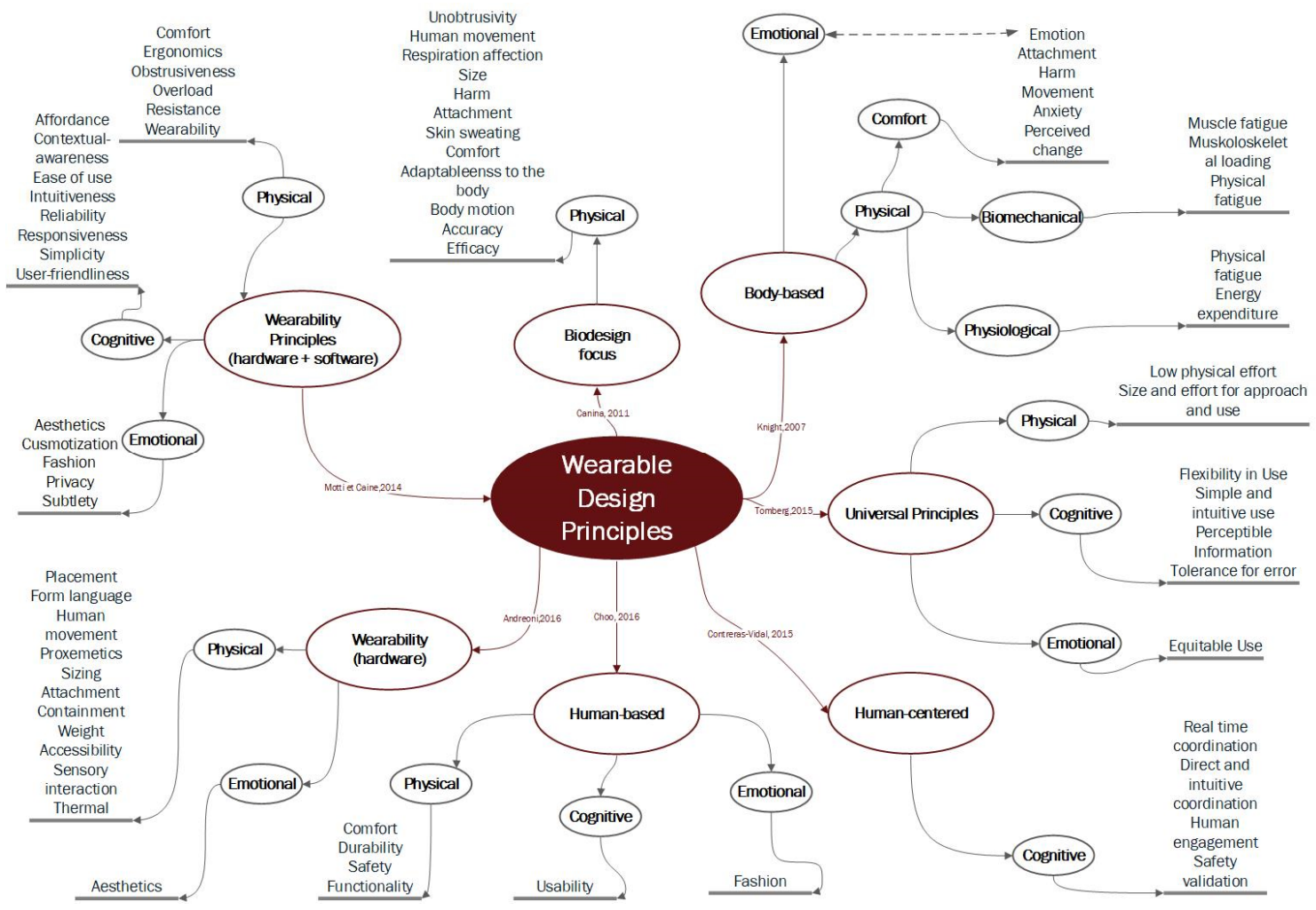

Figure 2. Studies that have considered human-factors in the definition of wearable design principles

\subsection{Quantitative analysis}

In order to analyse design requirements, it is important to consider not only the design requirements but also their definition. As previously mentioned, a single term for a design requirement can have different definitions in other studies. That means that in order to undertake an in-depth analysis, the definition should also be considered. To that end, this study has established the following criteria:

- Quantifying the number of times a specific design requirement is used in the studies $(\mathrm{N})$

- Quantifying the number of requirements inside the definition (P)

- Quantifying the number of times the requirement appears in another definition or requirement (C)

Table 1 shows the definitions considered in this study for the terms used above.

Table 1. Definition of the terms used for this study

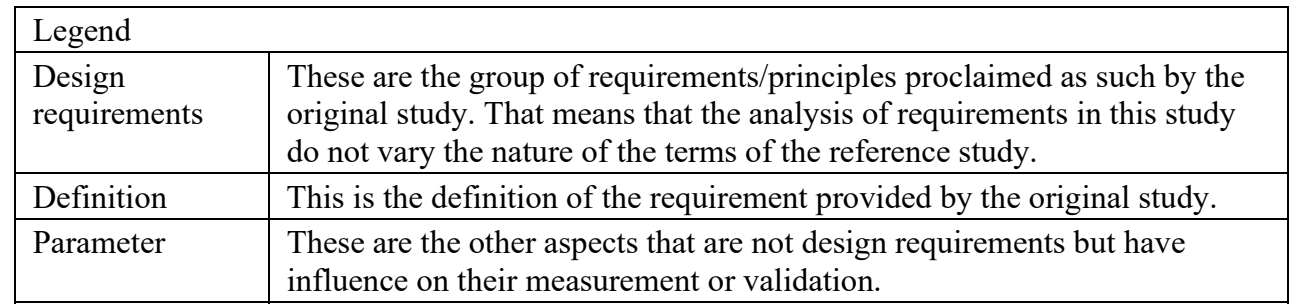


Figure 3 shows the requirements that appear in more than one study. As can be seen, "comfort" (4 out of 8 studies) is the most popular one, followed by "intuitiveness" ( 3 out of 8 studies) and "movement" ( 3 out of 8 ). The rest of the design requirements are used twice in the selected studies.

As mentioned in the previous section, this measurement is not enough to give reliable results due to the lack of the analysis semantics, the generality of the terms and the connection between different terms. For example, the term "comfort" is used in 4 different studies, and it in each one it has a different definition. In some studies it is used as a design requirement (Cho et al., 2009; Canina, 2011; Motti and Caine, 2014) and in others as a group of requirements (Knight et al., 2007). Furthermore, the term "comfort" is included in the definition of other design requirements, which means that a connection could be established. In analysing the rest of the design requirements, such as "obtrusiveness" or "sizing", it could also be concluded that they are included within the "comfort" group due to their meaning.

These conclusions led us to apply the criteria of quantifying the number of requirements inside the definition $(\mathrm{P})$ and quantifying the number of times the requirement appears in another definition or requirement $(\mathrm{C})$.

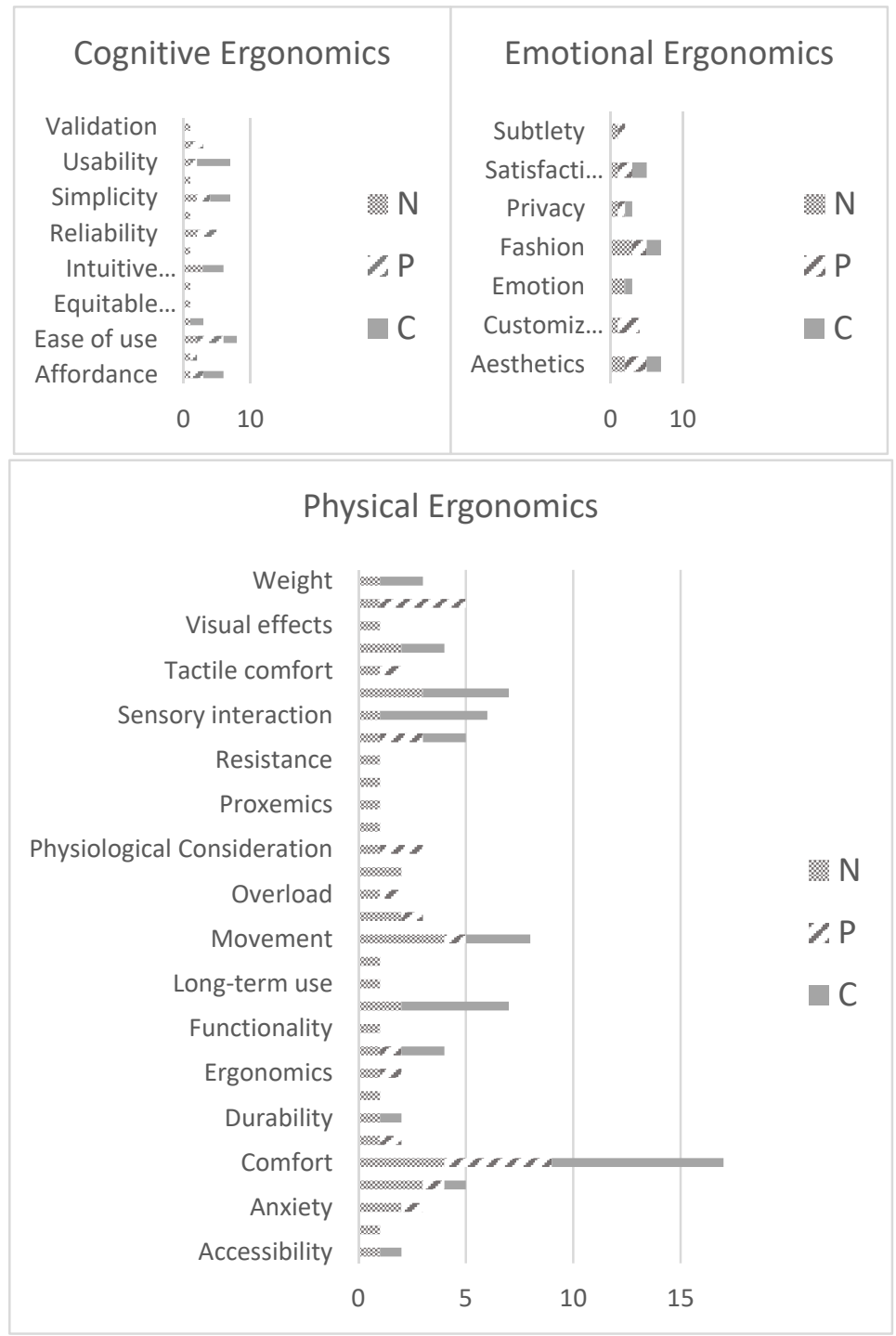

Figure 3. $\mathrm{N}, \mathrm{P}$ and $\mathrm{C}$ values in the list of design requirements found in the literature

If we go back to the term "comfort" as an example, we will see that inside its definition another design requirement mentioned in another study appears $(\mathrm{P}=1)$ : Comfort concerns the freedom from discomfort 
and pain. Users feeling enough comfort, no longer sense the device after wearing it for some time. Comfort involves an acceptable temperature, texture, shape, weight, and tightness. Comfortable devices fit users, enabling normal movements, without constraints. Flexible materials for instance, permit normal joint movements. Smaller form factors and more convenient sensor locations on the body can aid to ensure comfort. In this definition shows, the term "weight", which is considered a design requirement in other studies (Andreoni et al., 2016), is included. This means that weight is considered a critical factor when measuring comfort. Regarding the number of times "comfort" appears in another definition, it can be seen that there are eight definitions including comfort. This results show that "comfort" has a great relationship with other terms and definitions.

We found 9 different design requirements which include other requirements within their definition; "comfort" and "ease of use" are the most commonly used ones, followed by "simplicity" and "satisfaction". Secondly, there are 12 different requirements that appear in other definitions. Finally, the most common ones are "comfort" followed by "intuitiveness", "usability" and "movement". This leads to the conclusion that there is a high degree of interrelatedness and groupability in design requirements and their definition.

Figure 3 shows the quantification of the N, P and C parameters for the list of design requirements organized according to the three ergonomic domains.

This quantitative method helps to set the roadmap for establishing design requirements for wearables, but a qualitative phase is required to complete this first step. Indeed, the quantitative analysis informs us about the connectivity of different terms, but it does not contemplate the connection that two different requirements could have due to their meaning in its definition. Thus, the qualitative analysis criteria are detailed below.

\subsection{Qualitative analysis}

The aim of the qualitative methodology is to establish relationships between different design requirements. Doing so will make it easier to create groups and to analyse cause-effect relationships between different terms.

Two techniques have been used in this step: symmetric and asymmetric clustering matrixes, which are specific design tools for dealing with large amounts of data and showing interconnections (Kumar, 2012).

\subsubsection{Asymmetric clustering matrix}

The asymmetric clustering matrix is a design tool that compares two entities gathered during research and shows how each set breaks down into clusters based on its relation to the other set. It provides systematic analysis, facilitates comparison, reveals patterns and relationships and handles large sets of data, making it easy to visualize. The inputs are two sets of entities based on research findings and a matrix tool for scoring and sorting. The outputs are entity clusters based on the strength of the relations among them and insights about relations between two sets of entities.

In our case one entity is the six design requirements taken from (Cho et al., 2009), and the other is the 26 design requirements in which $\mathrm{N}$ or $\mathrm{P}$ or $\mathrm{C}>1$. Thus, the size of the matrix is 6 by 26 , and the scoring criteria are shown in Table 2.

Table 2. Matrix scoring criteria

\begin{tabular}{|l|l|l|}
\hline Score & Score Strength & Score description \\
\hline 0 & Weak relation & $\mathrm{N}$ or $\mathrm{P}$ or $\mathrm{C}$ parameters $>1$ \\
\hline 1 & Medium relation & Items are connected by $\mathrm{N}$ or $\mathrm{P}$ parameters \\
\hline 2 & Strong relation & Items are connected by $\mathrm{N}$ and $\mathrm{P}$ parameters \\
\hline
\end{tabular}

The result of the asymmetric clustering matrix is shown in Figure 4, which illustrates the 27 resulting relations.

Among the design requirements proposed by Cho (2019), the matrix shows that the item "comfort" has 10 relations with the rest of the design requirements proposed in the literature; five of them have a strong 
relation and five of them have a medium relation. The item "safety" has five relations, four of which are strong and two of which are medium. "Durability" has no relations with the rest of items and "functionality" has only two medium relations. "Usability" has seven relations, of which four are strong and three are medium. Finally, "fashion" has three relations, two of which are strong and one of which is medium.

These results show that the design requirements suggested by (Cho et al., 2009) do not include most of design requirements proposed by others authors, and that aspects such as "durability" or "functionality" have no relations or only very few relations.

Within the group of physical ergonomics, only "comfort", "safety" and "fashion" have a connection with the rest of the items. Regarding cognitive ergonomics, "comfort", "safety" and "usability" are the only ones that are connected. Finally, within the group of emotional ergonomics, "comfort", "functionality" and "fashion" are related but have few connections.

Consequently, although terms from different ergonomics are related, a common behaviour criterion cannot be established. In addition, this tool does not provide a solid road map for wearables design.

\begin{tabular}{|c|c|c|c|c|c|c|c|}
\hline & \begin{tabular}{|l}
$t$ \\
0 \\
0 \\
है \\
0
\end{tabular} & 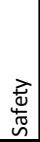 & 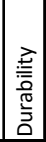 & 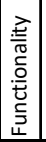 & 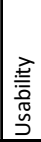 & 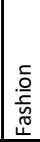 & \\
\hline Anxiety & 0 & 2 & 0 & 0 & 0 & 0 & \\
\hline Comfort & 2 & 0 & 0 & 0 & 0 & 1 & \\
\hline Form/shape & 1 & 1 & 0 & 0 & 0 & 0 & \\
\hline Movement & 2 & 0 & 0 & 0 & 0 & 0 & \\
\hline Obstrusiveness & 0 & 0 & 0 & 0 & 0 & 0 & 家 \\
\hline Perceived change & 0 & 0 & 0 & 0 & 0 & 0 & $\frac{\bar{\sigma}}{\frac{\pi}{\delta}}$ \\
\hline Safety & 0 & 2 & 0 & 0 & 0 & 0 & 気 \\
\hline Sizing & 0 & 0 & 0 & 0 & 0 & 0 & \\
\hline Wearability & 2 & 0 & 0 & 0 & 0 & 0 & \\
\hline Weight & 1 & 0 & 0 & 0 & 0 & 0 & \\
\hline Affordance & 0 & 0 & 0 & 0 & 0 & 0 & \\
\hline Ease of Use & 0 & 0 & 0 & 0 & 2 & 0 & \\
\hline \begin{tabular}{|l|} 
Engagement \\
\end{tabular} & 0 & 0 & 0 & 0 & 0 & 0 & שֶ \\
\hline Harm & 2 & 2 & 0 & 0 & 0 & 0 & है \\
\hline Intuitiveness & 0 & 0 & 0 & 0 & 1 & 0 & 은 \\
\hline PhysiologIcal consideration & 1 & 0 & 0 & 0 & 1 & 0 & 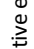 \\
\hline Realiability & 0 & 2 & 0 & 0 & 2 & 0 & 蓑 \\
\hline Simplicity & 0 & 0 & 0 & 0 & 1 & 0 & \\
\hline Usability & 0 & 0 & 0 & 0 & 2 & 0 & \\
\hline User-friendliness & 0 & 0 & 0 & 0 & 0 & 0 & \\
\hline Aesthetics & 0 & 0 & 0 & 1 & 0 & 2 & $\stackrel{\tilde{y}}{\underline{y}}$ \\
\hline Attachment & 2 & 0 & 0 & 0 & 0 & 0 & $\underline{\xi}$ \\
\hline Customization & 1 & 0 & 0 & 0 & 0 & 0 & 产 \\
\hline Emotion & 0 & 0 & 0 & 0 & 0 & 0 & $\overline{\bar{\pi}}$ \\
\hline Fashion & 1 & 0 & 0 & 0 & 0 & 2 & 总 \\
\hline Satisfaction & 0 & 0 & 0 & 1 & 2 & 0 & \\
\hline
\end{tabular}

Figure 4. Asymmetric clustering matrix

\subsubsection{Symmetric clustering matrix}

Based on the results of the asymmetric clustering matrix, a second matrix was proposed. The aim of the symmetric clustering matrix was to consider more items in order to achieve a higher amount of relations between design requirements. The design tool is similar to the asymmetric clustering matrix but it varies in size. In this case, design requirements with $\mathrm{N}$ or $\mathrm{P}$ or $\mathrm{C}>1$ were compared with each other, so the matrix was 26 squared. As it is a symmetric matrix, only one half is represented. 


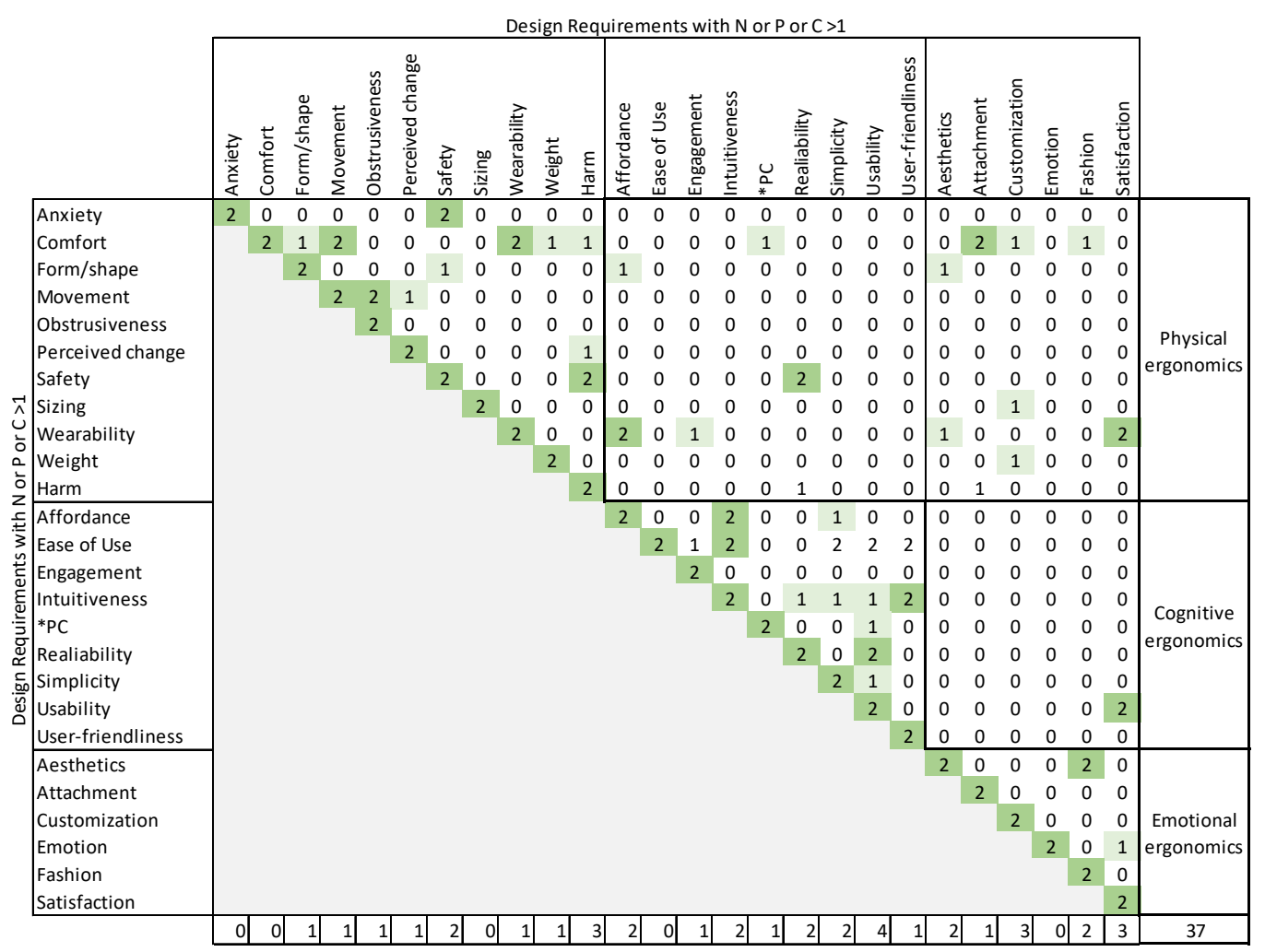

Figure 5. Symmetric clustering matrix

Figure 5 shows how the design requirements are related to each other. It resulted in 37 relations, which makes sense when taking into account that more items were used. Although the number of relations is much bigger than in the asymmetric clustering matrix, it can be seen that terms of same ergonomics are connected in a greater extent than terms of different ergonomic domains.

In the case of physical ergonomics, "comfort" has the highest number of relations followed by "form/shape", "safety", "wearability" and "harm". In terms of cognitive ergonomics, "intuitiveness" has the highest number of relations, followed by "usability" and "affordance". Finally, in the case of emotional ergonomics, only a few relations can be found: "aesthetics", "customization" and "satisfaction".

As can be seen, the results of this matrix are still not firm enough to draw a behaviour pattern.

Thus, based on the quantitative and qualitative analyses, the main conclusion is that there is not a common criterion for selecting and classifying the design requirements that should be considered in a wearable design process. There is a large list of requirements from different works related to human factors, but there is a clear gap in our knowledge about the relationships and hierarchy among them.

\section{A new framework}

Whenever the issue of wearables design is addressed, widespread doubts about the requirements that they should meet arise. It happens from the very beginning, when setting up a definition of wearables, as explained in the introduction. Multiple definitions of the term "wearable" exist, depending on the focus of each study. Some of them stay at the point of connectivity or other technical aspects in order to make them "fully-functional", "self-powered", and so on. As such, human aspects are often neglected, which can decrease not only users' acceptance of novel devices, but also their sustained engagement (Motti and Caine, 2014).

Some studies do add human factors and ergonomics in their definition of wearables, setting out a list of design principles to be integrated in the early stage of the design process. Thus, multiple definitions should be combined into a unique definition that considers both technical aspects and human factors. Clearly, this fact is directly related to the existence of different design requirements and, as was found in the literature review, there is a lack of common and clear criteria for defining such requirements. 
As a result of these studies, it is worth asking: Is it possible to reorganize all the requirements proposed in the literature and to unify their definitions? What are the dependency relationships among them? Are all these requirements at the same level? What would be the criteria for establishing the relationships among them? How can these parameters be measured or verified throughout the design process?

Given our quantitative and qualitative analysis of the aforementioned references, our structured analysis shows great ambiguity and a lack of organization and hierarchy of design requirements for wearable devices. Each author considers the requirements that he or she considers most relevant. But many questions that have no answer arise: Is there any structure that relates them together? Are all of them equally important and have to be considered at the same level? Must all of them be taken into account from the beginning of the design process, or can some come in during subsequent stages, such as the prototyping stage?

We propose a multi-level framework that organizes and relates these criteria. The Level 0 of this framework includes the three ergonomic domains (physical, cognitive, emotional). In the Level 1, the requirements have a more general character, because they include in their definitions other requirements, these are Comfort, Safety, Durability, Usability, Reliability, Aesthetics and Engagement. Comfort, according to Cho, concerns the freedom from discomfort and pain. Safety relates to being protected against physical, social, psychological or other types of harm. Durability concerns with the context of use and the experience of owning and using of the product. Usability, -as Nielson pointed out (Nielson 1993), has multiple components and is traditionally associated with learnability, efficiency, memorability, low error rate and satisfaction. Reliability refers to the level of confidence and trust that users have on the device. Aesthetics concerns aspects of the form and function of any wearable object, mainly associated with its attractiveness level and personal identity. Engagement refers with the privacy to exchange information, how transparent the communication is and should not disturb other people nearby.

Finally, in the Level 2 the requirements are divided in more detailed requirements, which can be measured with different methods, whether quantitative or qualitative. Moreover, in this framework some requirements from the studies analysed that have different names but similar meaning are unified; for example "ease of use" and "intuitiveness".

As Figure 6 shows, this multi-level framework includes 27 requirements, with 7 items in Level 1, and 20 in Level 2, which is a more manageable number of requirements for a design process than the initial 52 requirements taken from the proposed requirements in the analysed studies. Moreover, the weight of the design requirements from each ergonomics typology is more balanced than in the previous studies, where physical ergonomics was predominant. Consequently, this new framework reflects a more balanced contribution from all the ergonomics typologies to the final satisfaction of the user.

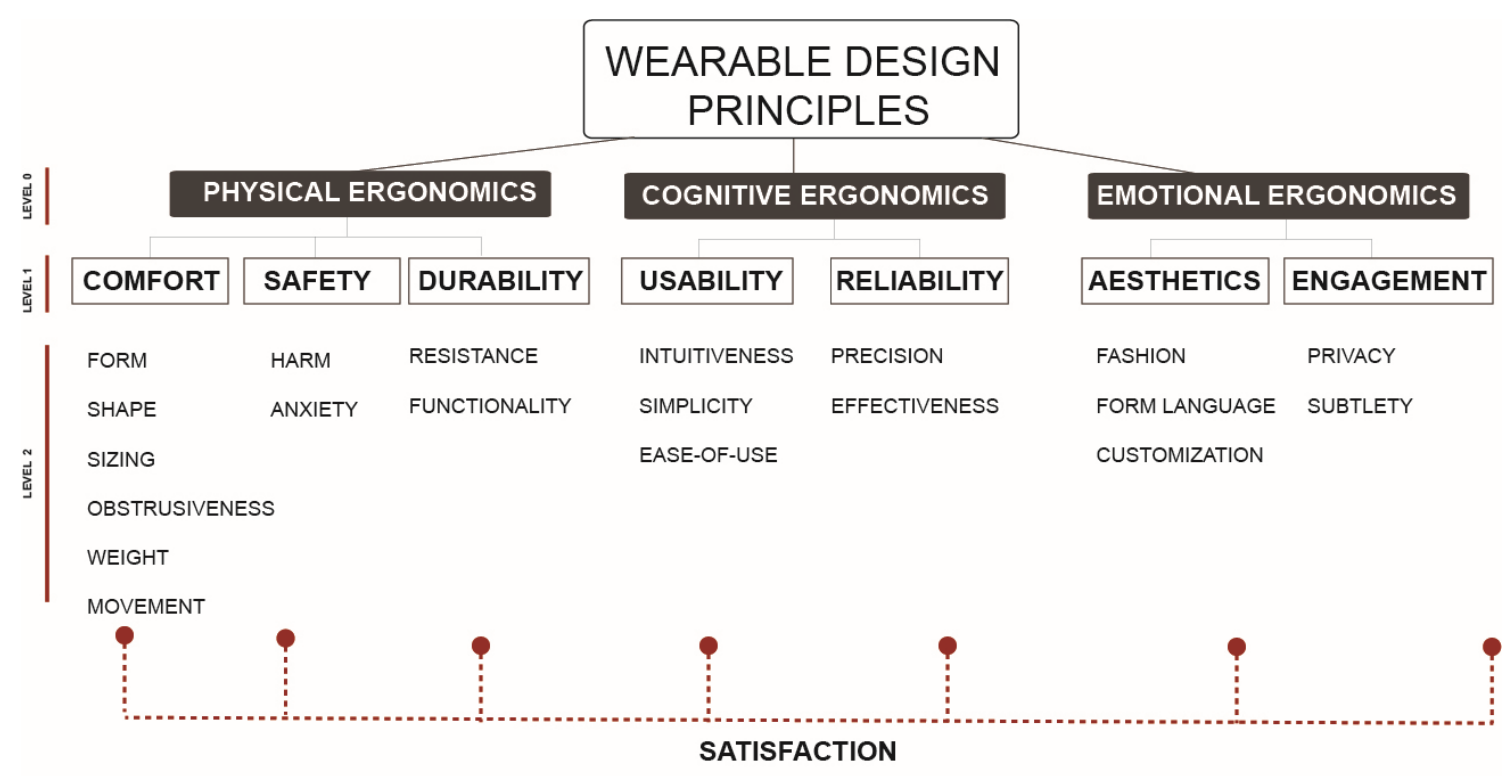

Figure 6. Wearable design requirements map 
According to the definition of a wearable device, there are two important requirements that need to be fulfilled in designing wearables: connectivity and wearability. That means that from the designer's point of view, the wearable, in addition to functioning properly, has to comply with a complex set of requirements to satisfy users in such a way that there will be long-term engagement. This framework aims to be a first step in the building of a solid framework for user-centred design of wearable devices, which will be further complemented and discussed with other researcher's point of view and experience.

\section{Discussion and conclusions}

Thus, this study had two objectives: (1) to review the design requirements found in the literature, and (2) provide a list of design requirements through the use of user-centred tools and techniques to design wearable devices.

According to the first objective, we surveyed the design requirements and found that the information follows a categorization scheme based on a reference model. This model was based on ergonomics and human factors. As a result of this exercise, it was seen that there were many more design requirements within the domain of physical ergonomics than in the domains of cognitive and emotional ergonomics. Moreover, the different use of language interfered in the analysis of each requirement's weight, so it was difficult to get a realistic picture of the current status of wearable design requirements. This fact led us to think that, although the general need to consider human factors is suggested in different works, there is a lot of confusion in the suggestion of requirements and the lack of human factors is not effectively addressed. This article presented a methodology to deal with these facts following the first steps of the methodology in Section 2.1 Ergonomics/human factors as a base for the methodology and in Section 2.2 Requirements visualization.

Regarding the second objective, a methodology for analysing design requirements was proposed. In this case, another reference model (Cho et al., 2009) was taken due to its reliable approach based on Maslow's hierarchy of needs. In this way, human factors were clearly considered. This reference model's requirements were compared to the most popular design requirement from the rest of studies, but no relevant results were obtained and thus a clear road map was not defined. In light of these results, a qualitative analysis was undertaken. In this case, apart from considering the terms we have also considered their definitions and the degree of relations they had. As a result of these analyses, some relations emerged, but they were not still helpful in defining a common pattern in the relation matrix. This process was done following the steps in Section 2.3 Quantitative analysis and Section 2.4 Qualitative analysis.

Finally, we presented a wearable design requirements map. In this step, certain criteria were specified, and the results were considered up to a point, but the lack of a reliable methodology remained. This map shown in the results helps to classify requirements in different levels so that it is easier to bear in mind the schema that should be followed. It also provides a solution where different terms are connected so it will be easier in the future to place them in specific stage of a design process.

By studying complete systems, ergonomics have to take on even greater importance and thus, they should be present in all the process stages. Not only should the physical part of the device be tested, but so should the interaction with the user. In order to consider this perspective, this study has suggested a categorization based on the three ergonomic domains: physical, cognitive and emotional. In this way, design requirements contain more aspects from the entire design cycle.

The goal of a design researcher is to create dialogue between the end users of a system, the broader stakeholders and the design team, and to illuminate the design issue in question. In this way, the role of ergonomics is critical in the design process of wearable prototypes since it is the point of connection between the tangible and intangible properties. Thus, wearable technology and wearable ergonomics should not be addressed independently.

Given the gaps pointed out in this paper, the following future research lines are proposed:

- Develop a methodology with final design requirements, including how (through prototypes or other design tools) and when (which stage of the design process) those requirements should be evaluated.

- Carry out the final validation of the above methodology through a real project in cooperation with a company or through a specific research project whose objective is to design a prototype of wearable. 
- Research on the identification of new parameters further from those included in the articles object of this study.

- Development of new tools, such as check lists or guides for designers, that can help designers to take into account all these parameters when they have to design a wearable.

\section{References}

Andreoni, G., Standoli, C.E. and Perego, P. (2016), "Defining Requirements and Related Methods for Designing Sensorized Garments”, Sensors, Vol. 16 No. 6, pp. 769. https://doi.org/10.3390/s16060769

Cambridge Dictionary (2017) [online] Cambridge Univ. Press. Available at: http://dictionary.cambridge.org/es/

Canina, M. and Ferraro, V. (2011), "A new approach in wearable devices: Biodesign Beyond the Boundaries", In: Chakrabarti, A. (Ed.), Research into Design - Supporting Sustainable Product Development, Research Publishing Services, pp. 283-291.

Chae, H.S., Hong, J.Y., Cho, H.S., Han, K.H., and Lee, J.H. (2007), “An investigation of usability evaluation for smart clothing", Proceedings of International Conference on Human-Computer Interaction, July 2007, Springer, Berlin, Heidelberg, pp. 1053-1060. https://doi.org/10.1007/978-3-540-73107-8_115

Chan, M., Estève, D., Fourniols, J.-Y., Escriba, C. and Campo, E. (2012), "Smart wearable systems: current status and future challenges", Artificial Intelligence in Medicine, Vol. 56 No. 3, pp. 137-156. https://doi.org/10.1016/j.artmed.2012.09.003

Cho, G., Lee, S. and Cho, J. (2009), "Review and Reappraisal of Smart Clothing”, In: Cho, G. (Ed.), Smart Clothing Technology and Applications, Taylor \& Francis Group, pp. 2-30. https://doi.org/10.1201/9781420088533-c1

Contreras-Vidal, J.L., Kilicarslan, A., Huang, H.H. and Grossman, R.G. (2015), "Human- Centered Design of Wearable Neuroprostheses and Exoskeletons", AI Magazine, Vol 36. No. 4, pp. 12-22. https://doi.org/10.1609/aimag.v36i4.2613

Duval, S., Hoareau, C. and Hiromichi, H. (2010), "Humanistic Needs as Seeds in Smart Clothing”, In: Cho, G. (Ed.), Smart Clothing Technology and Applications, Taylor \& Francis Group, CRC Press, pp. 153-189.

Knight, J.F., Deen-williams, D., Arvanitis, T.N., Baber, C., Sotiriou, S., Anastopoulou, S. and Gargalakos, M. (2007), "Assessing the Wearability of Wearable Computers", Wearable Computers, 10th IEEE International Symposium on 2006, IEEE, pp. 75-82.

Kumar, V. (2012), "Frame Insights", In: Kumar, V. (Ed.), 101 Design Methods, John Wiley\&Sons, pp. 158-165.

Motti, V.G. and Caine, K. (2014), "Human Factors Considerations in the Design of Wearable Devices", Proceedings of the Human Factors and Ergonomics Society Annual Meeting, Vol. 58 No. 1, pp. 1820-1824. https://doi.org/10.1177/1541931214581381

Nielson, J. (1993), Usability Engineering, AP Professional, Cambridge.

Sharples, S. and Wilson, J.R. (2014), "Methods in the Understanding of Human Factors", In: Wilson, J.R. and Corlett, N. (Eds.), Evaluation of Human Work, Taylor \& Francis Group, CRC Press, pp. 1-29.

Tomberg, V., Schulz, T. and Kelle, S. (2015), "Applying Universal Design Principles to Themes for Wearables", International Conference on Universal Access in Human-Computer Interaction, Springer, Cham, pp. 550-560. https://doi.org/10.1007/978-3-319-20681-3

Leire Francés-Morcillo, $\mathrm{PhD}$ Student

University of Navarra-Tecnun, Industrial Design Area

13 Manuel de Lardizábal St, 20018 Donostia-San Sebastián, Guipúzcoa, Spain

Email: pmorer@tecnun.es 